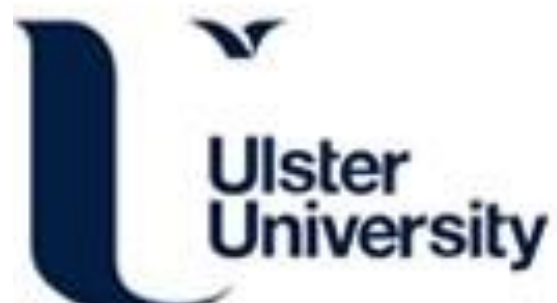

\section{Synergistic effects of hybrid conductive nanofillers on the performance of 3D printed highly elastic strain sensors}

Xiang, D., Zhang, X., Harkin-Jones, E., Zhu, W., Zhou, Z., Shen, Y., Li, Y., Zhao, C., \& Wang, P. (2019).

Synergistic effects of hybrid conductive nanofillers on the performance of 3D printed highly elastic strain

sensors. Composites Part A: Applied Science and Manufacturing. https://doi.org/j.compositesa.2019.105730

Link to publication record in Ulster University Research Portal

\section{Published in:}

Composites Part A: Applied Science and Manufacturing

\section{Publication Status:}

Published online: 16/12/2019

DOI:

j.compositesa.2019.105730

\section{Document Version}

Author Accepted version

\section{General rights}

Copyright for the publications made accessible via Ulster University's Research Portal is retained by the author(s) and / or other copyright owners and it is a condition of accessing these publications that users recognise and abide by the legal requirements associated with these rights.

\section{Take down policy}

The Research Portal is Ulster University's institutional repository that provides access to Ulster's research outputs. Every effort has been made to ensure that content in the Research Portal does not infringe any person's rights, or applicable UK laws. If you discover content in the Research Portal that you believe breaches copyright or violates any law, please contact pure-support@ulster.ac.uk. 


\section{Journal Pre-proofs}

Synergistic effects of hybrid conductive nanofillers on the performance of 3D printed highly elastic strain sensors

Dong Xiang, Xuezhong Zhang, Eileen Harkin-Jones, Wanqiu Zhu, Zuoxin Zhou, Yucai Shen, Yuntao Li, Chunxia Zhao, Ping Wang

PII: S1359-835X(19)30479-8

DOI: https://doi.org/10.1016/j.compositesa.2019.105730

Reference: JCOMA 105730

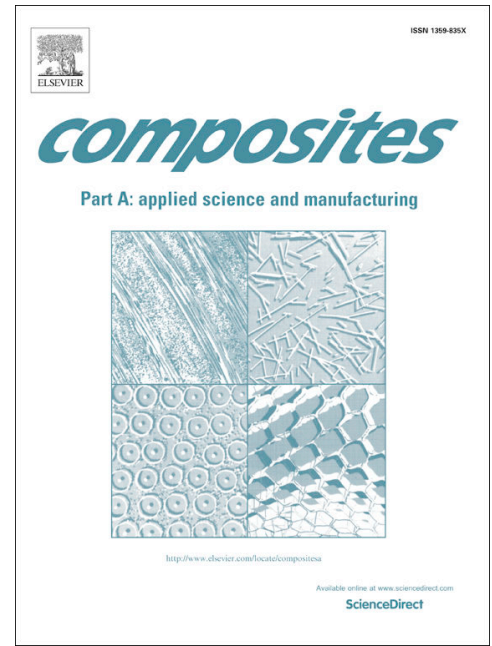

To appear in:

Composites: Part A

Received Date:

12 August 2019

Revised Date:

8 October 2019

Accepted Date:

10 December 2019

Please cite this article as: Xiang, D., Zhang, X., Harkin-Jones, E., Zhu, W., Zhou, Z., Shen, Y., Li, Y., Zhao, C., Wang, P., Synergistic effects of hybrid conductive nanofillers on the performance of 3D printed highly elastic strain sensors, Composites: Part A (2019), doi: https://doi.org/10.1016/j.compositesa.2019.105730

This is a PDF file of an article that has undergone enhancements after acceptance, such as the addition of a cover page and metadata, and formatting for readability, but it is not yet the definitive version of record. This version will undergo additional copyediting, typesetting and review before it is published in its final form, but we are providing this version to give early visibility of the article. Please note that, during the production process, errors may be discovered which could affect the content, and all legal disclaimers that apply to the journal pertain.

(C) 2019 Published by Elsevier Ltd. 


\section{Synergistic effects of hybrid conductive nanofillers on the performance of 3D printed highly elastic strain sensors}

Dong Xiang ${ }^{\mathrm{a},{ }^{*}, \# \text {, Xuezhong Zhang }}{ }^{\mathrm{a}, \# \text {, Eileen Harkin-Jones }}{ }^{\mathrm{b}}$, Wanqiu Zhu ${ }^{\mathrm{a}}$, Zuoxin Zhou $^{\mathrm{c}}$, Yucai Shen ${ }^{\mathrm{d}}$, Yuntao Li ${ }^{\mathrm{a},{ }^{*}}$, Chunxia Zhao ${ }^{\mathrm{a}}$, Ping Wang ${ }^{\mathrm{a}}$

aSchool of Materials Science and Engineering, Southwest Petroleum University, Chengdu 610500, China

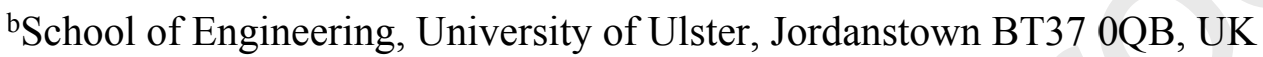

${ }^{c}$ Center for Additive Manufacturing, Faculty of Engineering, University of Nottingham, Nottingham NG7 2RD, UK

${ }^{\mathrm{d} C o l l e g e}$ of Materials Science and Engineering, Nanjing Tech University, Nanjing 210009, China

*Corresponding authors: dxiang01@hotmail.com (Dong Xiang); yuntaoli@swpu.edu.cn (Yuntao Li)

(\#These authors contributed equally to this work.)

Abstract: In this work, thermoplastic polyurethane based conductive polymer composites containing carbon nanotubes (CNTs) and synthesized silver nanoparticles (AgNPs) were used to fabricate highly elastic strain sensors via fused deposition molding. The printability of the materials was improved with the introduction of the nanofillers, and the size and content of the AgNPs significantly influenced the sensing performance of the 3D printed sensors. When the CNTs:AgNPs weight ratio was $5: 1$, the sensors exhibited outstanding performance with high sensitivity (GF $=43260$ at $250 \%$ strain), high linearity $\left(\mathrm{R}^{2}=0.97\right.$ within $50 \%$ strain $)$, fast response $(\sim 57 \mathrm{~ms})$, and excellent 
repeatability (1000 cycles) due to synergistic effects. A modeling study based on the Simmons' tunneling theory was also undertaken to analyze the sensing mechanism. The sensor was applied to monitor diverse joint movements and facial motion, showing its potential for application in intelligent robots, prosthetics, and wearable devices where customizability are usually demanded.

Keywords: synergy; strain sensor; 3D printing; nanocomposites

\section{Introduction}

In recent years, conductive polymer composites (CPCs) have been widely reported for the development of flexible strain sensors due to their excellent sensitivity, high elasticity, and large detectable range [1]. A variety of nanomaterials such as graphene [2-4], carbon nanotubes (CNTs) [5], metallic nanoparticles (NPs) [6], and metal nanowires have shown promising properties for the fabrication of CPCs [7,8]. Carbon nanoparticles with high aspect ratios, such as carbon nanotubes and graphene nanosheets are the most favorable conductive nanofillers when a low electrical percolation threshold is desired [9]. CNTs are a typical one-dimensional nanofiller that is prone to entangle, which facilitates the construction of a stable conductive network in the polymer matrix. Silver nanoparticles (AgNPs) play an important role in the field of microelectronics because of their good electrical conductivity. The surface and quantum size effects of AgNPs allow them to be used in applications such as surface-enhanced Raman and medical applications [10,11]. However, conductive nanofillers all have high surface energies and tend to agglomerate, which leads to poor dispersion in the polymer 
matrix.

To date, many studies have focused on improving the dispersion of the fillers by utilizing the synergistic effects between different nanofillers to construct a more robust conductive network $[12,13]$. This strategy has been extensively employed to establish reversible-transformable conductive networks, using carbon nanotubes, metal nanowires, graphene, and graphene foams, with the incorporation of various elastomers as the stretchable matrix $[14,15]$. For example, Liu et al. [16] obtained CPC with a low percolation threshold and tunable resistance strain sensing behavior via the addition of hybrid carbon nanotubes (CNTs) and graphene to thermoplastic polyurethane (TPU). The synergistic effects between the graphene and CNTs were identified using the excluded volume theory. Graphene acted as a "spacer" to separate the entangled CNTs from each other and the CNTs bridged the broad gap between the individual graphene sheets. This was beneficial for the dispersion of the CNTs and the formation of effective conductive paths, leading to better electrical conductivity with a lower conductive filler content. Cai et al. [17] studied the effects of filler types on the electrical and piezoresistive properties of conductive silicon rubber (SR) composites. Their work suggested that the synergistic effects of graphene and CB mixed fillers increased their dispersion in the SR matrix. Ma et al. [18] proposed a simple, low-cost and solution-based method for the preparation of a piezoresistive sensor based on conductive polyurethane (PU) sponges coated with synergistic multiwalled carbon nanotubes (MWCNTs) and reduced graphene oxide (RGO). Compared with the RGO@PU and MWCNT@PU sponges, the MWCNT/RGO@PU sponges demonstrated higher electrical conductivities because of 
the effective 3D MWCNT/RGO conducting network and superior sensing performance due to the synergistic effects of the diverse mechanisms. Sagalianov et al. [19] prepared a GNP/CNT/polymer nanocomposite based on solution mixing. They observed that the synergistic effects caused a significant decrease in the percolation threshold of the ternary polymer composites filled with carbon nanotubes and graphite nanoplatelets (GNPs) compared with the binary composite. Liu et al. [20] prepared a high-sensitivity graphene woven fabric (GWF)/PDMS composite sensor and connected it to a mobile phone via Bluetooth to create a wireless wearable device. Due to the flexible matrix, the composites show an excellent electrical conductivity arising from the CVD-grown graphenes, excellent stretchability and durability.

While the strain sensors reported in previous literature demonstrate good sensing performance, their fabrication is usually quite complicated and poorly suited to mass manufacturing. 3D printing (namely additive manufacturing) originated as a prototyping technology capable of making complex 3D parts at low cost and accurate physical reproduction [21] but this technology had advanced rapidly and is now being used as an actual manufacturing process rather than just for prototyping. Various printing techniques have been developed, which enables different types of materials to be used in $3 \mathrm{D}$ printing, such as metals by selective laser sintering [22], photocurable materials by stereolithography, inkjet printing [23], and thermoplastics by fused deposition modeling (FDM) [24]. Zhang et al. [25] used 3D printing to prepare porous polydimethylsiloxane (O-PDMS); it was integrated with carbon nanotubes and a graphene conductive network to obtain a highly ductile conductive material (OPCG). 
Compared with other 3D printing methods, FDM has the advantages of convenience, flexibility, and low cost, and the extruded filaments are suitable for large-scale production and also stable for long-term storage [25,26]. Gnanasekaran et al. [27] demonstrated the preparation, filament extrusion, and FDM-based 3D printing of CNT-based and graphene-based conductive polymer nanocomposites into functional 3D model structures. They demonstrated that the physical properties of the polymers, the dispersion, and the aspect ratio of the conductive fillers play important roles in the evolution of conducting networks. Kim et al. [14] used FDM to fabricate a multi-axial force sensor, which was consisted of the TPU structural component and the CNT/TPU nanocomposite sensing component. However, most strain sensors that are currently produced are unable to satisfy all the performance parameters (including a gauge factor above 15 within a strain of $50 \%$, a wide detectable strain range of $0-250 \%$, and a linearity close to 1 ), in combination with low-cost production and a printability.

In this work, AgNPs were synthesized by electrophoretic deposition in the presence of CNTs for better dispersion. The ternary CNT/AgNP/TPU nanocomposites were prepared by solution mixing and extruded into filaments using a single-screw extruder for subsequent printing into flexible strain sensors by FDM. The AgNPs in this study prevent the intact interaction between neighboring CNTs as well as tailoring the conductive pathways in the composite. The performance of the printed sensors, including the gauge factor, detectable strain range, and linearity was mainly regulated by the amount of silver nanoparticles present. To the best of author's knowledge, there is almost no literature focusing on the synergistic effect of hybrid conductive nanofillers 
in $3 \mathrm{D}$ printed flexible strain sensors to date. This work also provides important guidance for the facile fabrication of flexible and high-performance strain sensors via the 3D printing technique where complex designs, multi-directionality, and customizability are usually demanded.

\section{Experimental section}

\subsection{Materials}

Multi-walled carbon nanotubes (NC7000) were obtained from Nanocyl SA (Belgium). The CNTs had a nominal average length of $1.5 \mu \mathrm{m}$ and a nominal diameter of $9.5 \mathrm{~nm}$. The TPU powder (Bayer 2195) was supplied by Bayer Co. Ltd. with a melt flow index of $17.5 \mathrm{~g} / 10 \mathrm{~min}\left(215{ }^{\circ} \mathrm{C}\right.$, at a pressure of $\left.10 \mathrm{~kg}\right)$ and a density of $1.12 \mathrm{~g} / \mathrm{cm}^{3}$. Dimethylformamide (DMF) reagent, polyvinylpyrrolidone (PVP, K-30), and silver nitrate (97\% purity) were purchased from Chengdu Kelong Chemical Reagent co. Ltd. Sodium citrate was supplied by Tianjin Zhiyuan Chemical Reagent co. Ltd.

\subsection{Synthesis of CNT/AgNP nanofillers}

The CNT/AgNP hybrid nanofillers with different CNT:AgNP weight ratios (10:1, 5:1 and 2:1) were synthesized. For the CNT/AgNP (10:1) sample, a flask with silver nitrate aqueous solution (AR, $0.12 \mathrm{mM}$ ) was heated in an oil bath at $120^{\circ} \mathrm{C}, 1 \mathrm{mg}$ of CNTs and

$10 \mathrm{~mL}$ sodium citrate aqueous solution (AR, $1 \mathrm{wt} \%$ ) were added into the flask sequentially, as shown in Fig. 1a. The solution turned grey after stirring while heating in the oil bath at $120^{\circ} \mathrm{C}$ for $1 \mathrm{~h}$. The CNT/AgNP (10:1) sample was centrifuged at 10,000 rpm (KH-250DE, Kunshan, China) and stored in absolute ethanol for use. Likewise, the 
content of raw materials for preparing the CNT/AgNP(5:1), CNT/AgNP(2:1) and

AgNPs nanofillers is shown in Table 1. Sufficient nanofillers were synthesized

following this method for the subsequent preparation of nanocomposites. Table 1.

Content of raw materials for preparing CNT/AgNP and AgNP nanofillers.

\begin{tabular}{cccc}
\hline Samples & CNTs (mg) & $\mathrm{AgNO}_{3}(\mathrm{mM})$ & sodium citrate $(\mathrm{mL}, 1 \mathrm{wt} \%)$ \\
\hline $\mathrm{CNTs}$ & 1 & & 10 \\
$\mathrm{CNT} / \mathrm{AgNP}(10: 1)$ & 1 & 0.12 & 20 \\
$\mathrm{CNT} / \mathrm{AgNP}(5: 1)$ & 1 & 0.22 & 45 \\
$\mathrm{CNT} / \mathrm{AgNP}(2: 1)$ & 1 & 0.53 & 10 \\
AgNPs & - & 0.12 & \\
\hline
\end{tabular}

\subsection{Preparation of nanocomposites}

First, the CNTs or hybrid CNT/AgNP nanofillers were dispersed in DMF via ultra-sonication at $100 \mathrm{~W}$ and $40 \mathrm{kHz}$ for $1 \mathrm{~h}$ at room temperature. TPU powder was dissolved in the resulting suspension by magnetic stirring for $2 \mathrm{~h}$. The total content of the nanofillers in all test samples was $3 \mathrm{wt} \%$. The mixed suspension was transferred into a glass container $(140 \mathrm{~mm} \times 140 \mathrm{~mm} \times 40 \mathrm{~mm})$ to dry for $24 \mathrm{~h}$ at $80{ }^{\circ} \mathrm{C}$ in an air-circulating oven to produce the nanocomposite sheets. Nanocomposites without the AgNPs were also prepared by the same method for comparison (Fig. 1b).

\subsection{D printing of strain sensors}

The nanocomposite sheets were cut into small granules and extruded into filaments with a diameter of $1.75 \mathrm{~mm}$ using a benchtop single-screw extruder (Wellzoom Type 
C). The operating temperature was $190{ }^{\circ} \mathrm{C}$ and the screw speed was $100 \mathrm{rpm}$. The filament was then used to print strain sensors using an ET-K1 (ET Co. Ltd., China) desktop FDM 3D printer. A stacking mode was used for the 3D printing with an interlayer angle of $90^{\circ}$ (Fig. 1c). The FDM printing parameters were optimized for improved printability and are shown in Table 2. All the samples used for the tensile, electrical, and electromechanical tests were printed under the same conditions. The material strips had a dimension of $50 \mathrm{~mm} \times 10 \mathrm{~mm} \times 1 \mathrm{~mm}$.

(a)

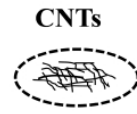

$\mathrm{AgNO}_{3}$



(b)

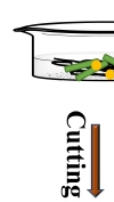

(c)
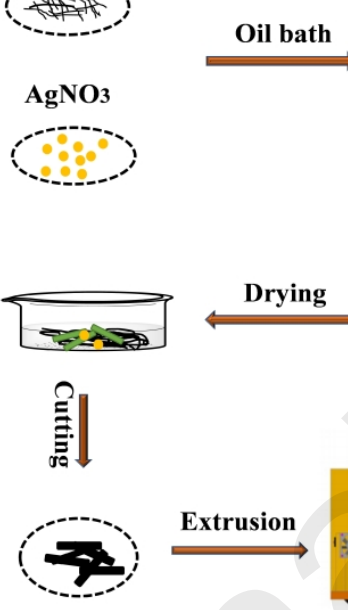

$\mathrm{CNT} / \mathrm{AgNP} / \mathrm{H}_{2} \mathrm{O}$
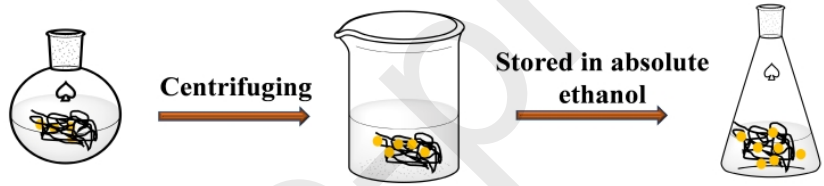

CNT/AgNP/TPU/DMF
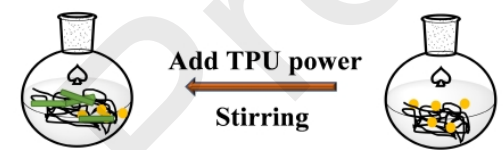

Ultrasonicating
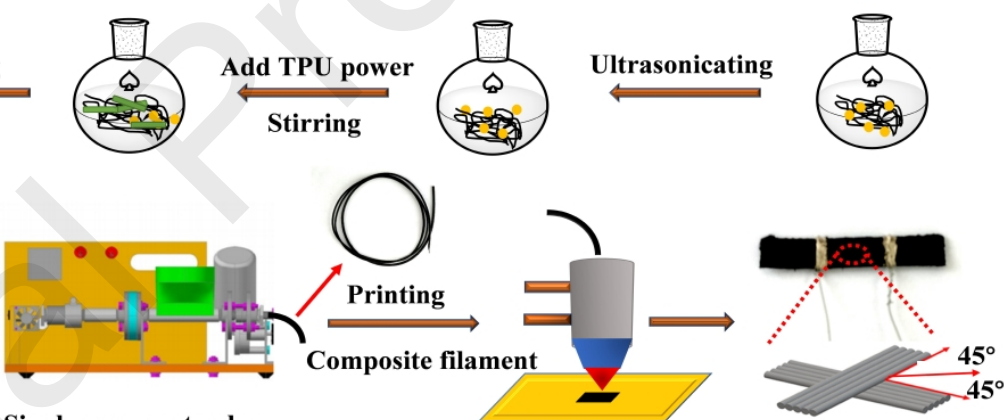

Single screw extruder

Fig 1. Schematic of the 3D printed strain sensor: (a) preparation of a mixture of CNTs and AgNPs, (b) preparation process for the modified CNT/TPU nanocomposite film, (c) preparation of the 3D printed nanocomposites.

Table 2. 3D printer operating parameters.

\begin{tabular}{cc}
\hline Printing parameters & Values \\
\hline Layer thickness $(\mathrm{mm})$ & 0.2 \\
Nozzle temperature $\left({ }^{\circ} \mathrm{C}\right)$ & 220 \\
\hline
\end{tabular}


Hot bed temperature $\left({ }^{\circ} \mathrm{C}\right)$

Printing speed $(\mathrm{mm} / \mathrm{s})$

Filling rate $(\%)$

100

Nozzle diameter (mm)

0.4

\subsection{Characterization}

\subsubsection{SEM and EDS}

The morphologies of CNTs and AgNPs hybrid nanofiller were investigated using a ZEISS EV0 MA15 scanning electron microscope (SEM) after ultrasonication and vacuum drying. The elemental composition of the CNTs and AgNPs were confirmed via energy-dispersive X-ray spectroscopy (EDS, Oxford Instruments X-MaxN). The morphologies of the printed nanocomposites were examined by FESEM (FEI Quanta 650 FEG) using an accelerating voltage of $20 \mathrm{kV}$. The specimens were cryogenically fractured in liquid nitrogen perpendicular to the axial direction. Then, the fractured section was directly observed without gold sputtering.

\subsubsection{Electrical resistivity measurement}

The volume electrical resistivity $(\rho)$ of the nanocomposites was measured in the cross-layer (longitudinal) and through-layer (through-thickness) directions using a two-point method combined with a picoamp-meter (Keithley 6485) and a DC digital source meter (Tektronix PWS4323) at $3 \mathrm{~V}$. The electrode distances were 15 and $1 \mathrm{~mm}$ in cross-layer and through-layer directions respectively (Fig. 2a). Silver paste was used 
to minimize contact resistance between the specimen and electrodes. The resistivity of the nanocomposite films was calculated using Eq. (1):

$$
\rho=\mathrm{R} \frac{S}{L_{0}}
$$

where $\mathrm{R}$ is the electrical resistance of the sample, and $L_{0}$ and $S$ are the electrode distance and cross-sectional area of the sample in testing direction, respectively.

\subsubsection{Electromechanical testing}

Characterization of the electromechanical performance of the printed samples was carried out by measuring the resistance change under monodirectional and cyclic (stretching/releasing) loading using an MTS CMT4104 universal tester combined with a picoamp-meter and a digital source meter at a fixed voltage of $3 \mathrm{~V}$ (Fig. 2b). The samples used for electromechanical performance testing were identical to those for the electrical resistivity measurements.

The gauge factor (GF) was calculated using Eq. (2) based on the relative resistance change $\left(\Delta R / R_{0}\right)$ and the tensile strain:

$$
\mathrm{GF}=\frac{\Delta R}{R_{0} \times \varepsilon}
$$

where $R_{0}(\Omega), \Delta R(\Omega)$, and $\varepsilon(\%)$ represent the initial resistance without strain, resistance change with strain, and the applied tensile strain, respectively. 
(a)

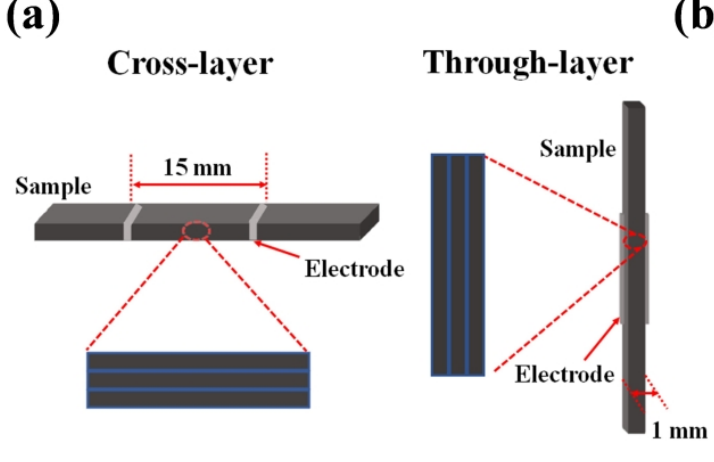

(b)

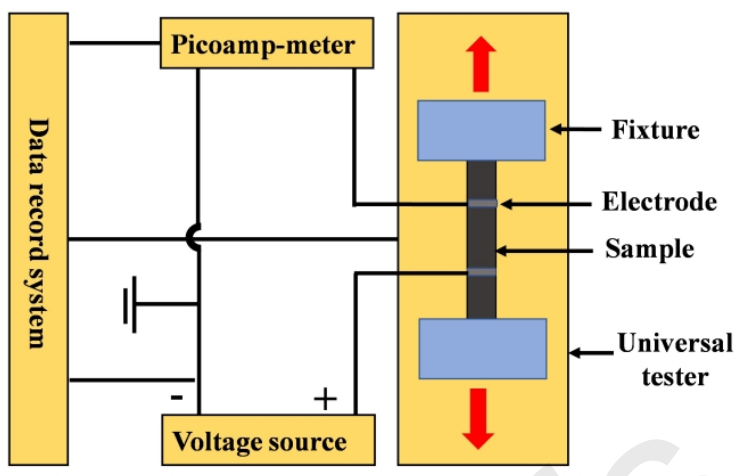

Fig 2. Schematic diagram of (a) electrical resistivity measurements in cross-layer and through-layer directions and (b) strain sensing tests.

\section{Results and Discussion}

\subsection{Printability}

The critical buckling pressure $\left(P_{c r}\right)$ of the filament in the FDM printer can be calculated using the Euler buckling equation [28]:

$$
P_{c r}=\frac{\pi^{2} E D_{f}^{2}}{16 L_{f}^{2}}
$$

where $E$ is the elastic modulus of the filament, $D_{f}$ is the diameter of the filament, and $L_{f}$ is the length of filament from the drive gear to the melting zone. If the filament is not sufficiently stiff then it will buckle in the FDM machine and printing will stop (the critical buckling pressure of the filament must be higher than the pressure required to extrude the polymer through the nozzle). Since TPU is a relatively low modulus material which may be prone to buckling it is beneficial to enhance its modulus to improve printability. From Fig. 3a, b, and Table 3, it can be seen that the addition of nanofillers increases the elastic modulus and buckling resistance of the filaments. In particular, the amount of silver nanoparticles used had a significant effect 
on the elastic modulus of the composite filaments. Compared with CNT/TPU, as the ratio of CNTs to AgNPs increases to 5:1, the elastic modulus and critical buckling pressure of the composite filament increase by $60.9 \%$ and $60 \%$, respectively, which improved the printability of the composite filament. However, on further increasing the AgNPs content, the strengthening effect of the material by AgNPs was much lower than by the CNTs, leading to a decrease in the elastic modulus and critical buckling pressure of the composite filament $\left(\mathrm{E}=24.2 \pm 1.16 \mathrm{MPa}, P_{c r}=9.3 \pm 0.47 \mathrm{KPa}, \mathrm{CNT}\right.$ : AgNP $=2: 1$ ). This can be attributed to the poorer dispersion of nanofillers and will be discussed in the following section.
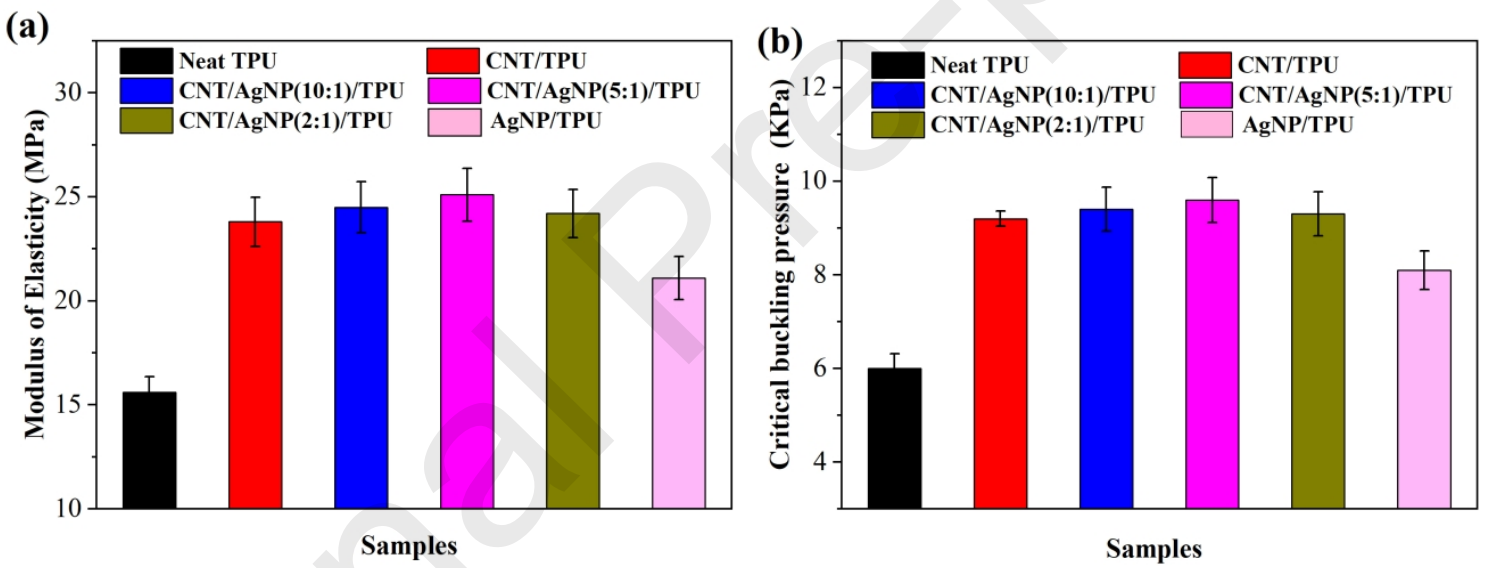

Fig. 3. Modulus of elasticity (a) and critical buckling pressure (b) of the extruded filaments with different nanofiller composition.

Table 3. Changes in the modulus of elasticity and critical buckling pressure of filaments with different nanofiller composition.

\begin{tabular}{ccccc}
\hline Samples & $\mathrm{E}(\mathrm{MPa})$ & $\Delta \mathrm{E}(\%)$ & $\mathrm{P}_{\mathrm{cr}}(\mathrm{KPa})$ & $\Delta \mathrm{P}_{\mathrm{cr}}(\%)$ \\
\hline Neat TPU & $15.6 \pm 0.75$ & -- & $6.0 \pm 0.31$ & -- \\
CNT/TPU & $23.8 \pm 1.18$ & $52.6 \%$ & $9.2 \pm 0.46$ & $53.3 \%$ \\
CNT/AgNP(10:1)/TPU & $24.5 \pm 1.22$ & $57.1 \%$ & $9.4 \pm 0.47$ & $56.7 \%$
\end{tabular}




$\begin{array}{ccccc}\text { CNT/AgNP(5:1)/TPU } & 25.1 \pm 1.26 & 60.9 \% & 9.6 \pm 0.48 & 60.0 \% \\ \text { CNT/AgNP(2:1)/TPU } & 24.2 \pm 1.16 & 55.1 \% & 9.3 \pm 0.47 & 55.0 \% \\ \text { AgNP/TPU } & 21.1 \pm 1.03 & 35.3 \% & 8.1 \pm 0.41 & 35.0 \%\end{array}$

\subsection{Morphology}

The morphologies of the CNTs and AgNPs were firstly observed using SEM. The addition of CNTs in the reaction process is equivalent to providing a nucleating agent for the AgNPs, which reduced the size of the AgNP crystals while increasing the reaction rate $[29,30]$. Also, the AgNPs act to prevent interactions between the neighboring CNTs, which reduces CNT agglomeration. From Fig. 4a, it is clear that the AgNPs are not only well distributed in the CNTs but also have a small size (the diameter of the AgNPs is no more than $100 \mathrm{~nm}$ ). From Fig. 4b, the size of the formed AgNPs slightly increased because of the decrease in the amount of CNTs, and the AgNPs remained well distributed in the CNTs. In this case, the silver nanoparticles are spherical and less than $150 \mathrm{~nm}$ in diameter. From Fig. 4c, the AgNPs show obvious agglomeration in the CNTs. Additionally, numerous AgNPs with sizes above $300 \mathrm{~nm}$ can be observed; its morphology is mostly lamellar or flaky, which is mainly attributed to the silver nanoparticles being highly unstable and tending to aggregate together or undergo Oswald maturation $[31,32]$.

EDS analysis was conducted for the samples shown in Fig.4a, b, and c respectively. Fig. 5 shows the EDS results for the elemental analysis. The strong EDS signals can be attributed to the hybrid nanofillers and also used to examine the chemical composition of the nanomaterials $[31,33]$. The EDS spectrum in Fig. 5 consists of peaks for carbon, 
silver, oxygen, sodium, and silica, while the peaks for oxygen, sodium, and silica are very weak. The amount of silver increases as the amount of CNTs decreases during the AgNPs nucleation reaction. As expected, the ratios of the carbon and silver elements are close to 10,5 , and 2 , respectively.

A clear multi-layer structure with a thickness of $\sim 200 \mu \mathrm{m}$ for each layer can be seen in the printed nanocomposites (Fig. 4d). Additionally, there were some voids in the printed samples despite the good interlayer adhesion. The dispersion of the CNTs and AgNPs in the TPU matrix is shown in Fig. 4e and Fig. 4f. When the cross-section of sample was irradiated by the SEM, unlike CNT/TPU, the CNT/AgNP/TPU was significantly brighter, indicating that the CNT/AgNP/TPU had a higher conductive path density than CNT/TPU. The bright white spots are attributed to the conductivity of the AgNPs, which is higher than the CNTs. In Fig. 4e, a large number of clustered CNTs were observed. This is mainly due to the strong van der Waals force between the CNT molecules, which caused formation of loose agglomerates. Fig. $4 \mathrm{f}$ shows that the CNT and AgNP nanofillers are uniformly dispersed. When the AgNPs were added between the CNTs, they form a hierarchical CNT/AgNP system that inhibits the agglomeration of CNTs, thereby producing a good dispersion. 



Fig. 4. SEM micrographs of the CNT/AgNP nanofillers and the cross-sections of nanocomposites: (a) CNT/AgNP (10:1), (b) CNT/AgNP (5:1), (c) CNT/AgNP (2:1), red circles indicate the AgNPs, (d-e) CNT/TPU, (f) CNT/AgNP (5:1)/TPU. It should be noted that the specimens in (e) and (f) are not gold sputtered to observe the conductive network based on the rich secondary electrons emitted from the conductive nanofillers.

Fig. 5. Energy dispersive spectrum of hybrid nanofillers: (a) CNT/AgNP (10:1),

(b) CNT/AgNP (5:1), (c) CNT/AgNP (2:1).

\subsection{Electrical resistivity measurement}

Changes in the resistivity of the nanocomposites with different hybrid nanofillers is shown in Table 4. In the cross-layer direction, the resistivity of the printed sample is nearly 7 times lower than that of the through-direction. This can be attributed to the slight orientation of the CNTs during extrusion and 3D printing, which results in the reorganization of the conductive network structure with a preferential alignment in the extrusion/print direction and thus higher conductivity in that direction [34]. The resistivity of the printed samples decreases first and then increases with increasing AgNP content. In the cross-layer direction, the resistivity of the CNT/TPU is $3.17 \times 10^{4}$ $\Omega \cdot \mathrm{m}$, the resistivity of $\mathrm{CNT} / \mathrm{AgNP}(5: 1) / \mathrm{TPU}$ with a weight ratio of $5: 1$ between the CNTs and AgNPs is $1.23 \times 10^{4} \Omega \cdot \mathrm{m}$, and the resistivity of the AgNP/TPU is as high as $5.36 \times 10^{5} \Omega \cdot \mathrm{m}$. The resistivity of the printed sensors initially decreases with the addition of AgNPs for the following reasons: first, the conductivity of AgNPs is better than that of CNTs [35]; second, the silver nanoparticles prevent agglomeration of neighboring carbon nanotubes, facilitating the formation of conductive pathways [6,14]. Fig. 6a shows that it is difficult to control the intertwining between CNTs. However, the synergistic effects between AgNPs and CNTs (weight ratio 5:1) further complement the conductive network in the polymer matrix (Fig. 6b) [36,37]. On increasing the amount of $\mathrm{AgNO}_{3}$, the AgNPs increase in size due to agglomeration, resulting in an easily collapsible, less-packed conductive network inside the elastomer matrix, as shown in 
Fig. 6c. In Fig. 6d, the high resistivity of the AgNP/TPU composite may be mainly attributed to the significant agglomeration of AgNPs during the reaction, which reduced the dispersion of AgNPs in TPU.

Table 4. Electrical resistivity of the 3D printed strain sensors.

\begin{tabular}{ccc} 
Samples & \multicolumn{2}{c}{ Resistivity $(\Omega \cdot \mathrm{m})$} \\
\cline { 2 - 3 } & Cross-layer & Through-layer \\
\hline $\mathrm{CNT} / \mathrm{TPU}$ & $3.17 \times 10^{4}$ & $2.01 \times 10^{5}$ \\
$\mathrm{CNT} / \mathrm{AgNP}(10: 1) / \mathrm{TPU}$ & $2.01 \times 10^{4}$ & $1.21 \times 10^{5}$ \\
$\mathrm{CNT} / \mathrm{AgNP}(5: 1) / \mathrm{TPU}$ & $1.23 \times 10^{4}$ & $8.34 \times 10^{4}$ \\
$\mathrm{CNT} / \mathrm{AgNP}(2: 1) / \mathrm{TPU}$ & $4.74 \times 10^{4}$ & $3.29 \times 10^{5}$ \\
$\mathrm{AgNP} / \mathrm{TPU}$ & $5.36 \times 10^{5}$ & $3.75 \times 10^{6}$ \\
\hline
\end{tabular}


AgNP

TPU 
Fig. 6. Schematic diagram of CNT/AgNP network in nanocomposites: (a), (e)

CNT/TPU; (b), (f) CNT/AgNP(5:1)/TPU; (c), (g) CNT/AgNP(2:1)/TPU; (d), (h) AgNP/TPU.

\subsection{Electromechanical performance}

All the 3D printed sensors exhibited a steady increase in resistance under monodirectional loading, indicating a clear electromechanical response. Generally, better nanofillers dispersion contributed to improvements in the sensitivity and linearity of the strain sensors. It can be observed in Fig. 7 that the CNT/TPU strain sensors have the lowest sensitivity $(\mathrm{GF}=2.97$ at a strain of $50 \%)$ and linearity $\left(\mathrm{R}^{2}=0.83\right)$ of all the printed samples, which can be attributed to the numerous CNT agglomerates in the TPU matrix (Fig. 6e). These results indicate that the use of CNTs alone may not be an appropriate strategy for the fabrication of high-sensitivity strain sensors. In fact, the CNT-based strain sensors reported to date have generally demonstrated limited sensitivity $[38,39]$. With the addition of AgNPs, the sensitivity and linearity of the sensor is significantly improved. Upon the introduction of a small amount of AgNPs at a CNTs:AgNPs weight ratio of 10:1, the gauge factor (GF $=5.49$ at a strain of $50 \%)$ and linearity $\left(\mathrm{R}^{2}=0.91\right)$ of the sensor simultaneously increase. When the weight ratio of CNTs: $\operatorname{AgNPs}$ is $5: 1$, the gauge factor $(\mathrm{GF}=15.2$ at a strain of $50 \%)$ and linearity $\left(\mathrm{R}^{2}=\right.$ 0.97) further increase. This can be explained by the synergy between the CNTs and AgNPs in terms of spatial distribution of each NP with the AgNPs acting as a spacer that prevents interaction between neighboring CNTs as well as tailoring the conductive pathways (Fig. 6f). When the amount of AgNPs relative to CNTs is further increased 
(ratio of CNTs:AgNPs is 2:1) the sensitivity of the sensor decreases significantly (GF $=$ 8.01 at a strain of $50 \%$ ). This behavior may be attributed to an increased amount of AgNP agglomerates [40]. However, the linearity of this sample slightly increased $\left(\mathrm{R}^{2}=\right.$ 0.99), which might be associated with the limited presence of free carbon nanotubes in the composite (Fig. 6g) [14]. It should be noted that because of the poor conductivity of the AgNP/TPU composites with increased AgNP agglomeration (Fig. 6h), the printed sensor lost its response signal at small strains, thus it is not shown in this paper. At a strain of $250 \%$, the GF values for the sensors based on CNT/TPU, CNT/AgNP(10:1)/TPU, CNT/AgNP(5:1)/TPU, and CNT/AgNP(2:1)/TPU are 1389, 2653, 43260, and 12396 respectively. The non-linear behavior of the sensors at strain levels above $50 \%$ is related to the non-linear strain-sensitivity of the matrix polymer [41]. When the strain exceeds $250 \%$, the conductive network in the sensors was significantly damaged, resulting in a rapid increase in the $\Delta R / R_{0}$ of the strain sensor (the response signal is hard to be monitored). Therefore, the maximum detectable range of the sensors is about $250 \%$ strain.

In general, the printed $\mathrm{CNT} / \mathrm{AgNP}(5: 1) / \mathrm{TPU}$ sensors exhibit excellent performance in terms of sensitivity, linearity, and detectable strain range compared with other recently reported strain sensors (see Fig. 8)[1,4,5,8,14,20,41-47]. Fig. 9 shows the instant response of the sensors. The lowest response time $(57 \mathrm{~ms})$ for the printed sensor occurs when CNT:AgNP $=5: 1$. This short response time is superior to that reported for many flexible sensors [42-48]. By way of example, Ge et al. [48] prepared a flexible strain sensor with a relatively low response time of $75 \mathrm{~ms}$ using $\mathrm{rGO} /$ polyaniline 
wrapped polyurethane sponge. The very low response time for CNT/AgNP (5:1)/TPU strain sensor can be attributed to the following two aspects: first, the strain sensor has high sensitivity to accurately recognize minute strain, reducing time delay at the initiation of deformation; second, the addition of AgNPs improves the dispersion of CNTs in the polymer matrix, which is beneficial to the rapid response of the sensor.

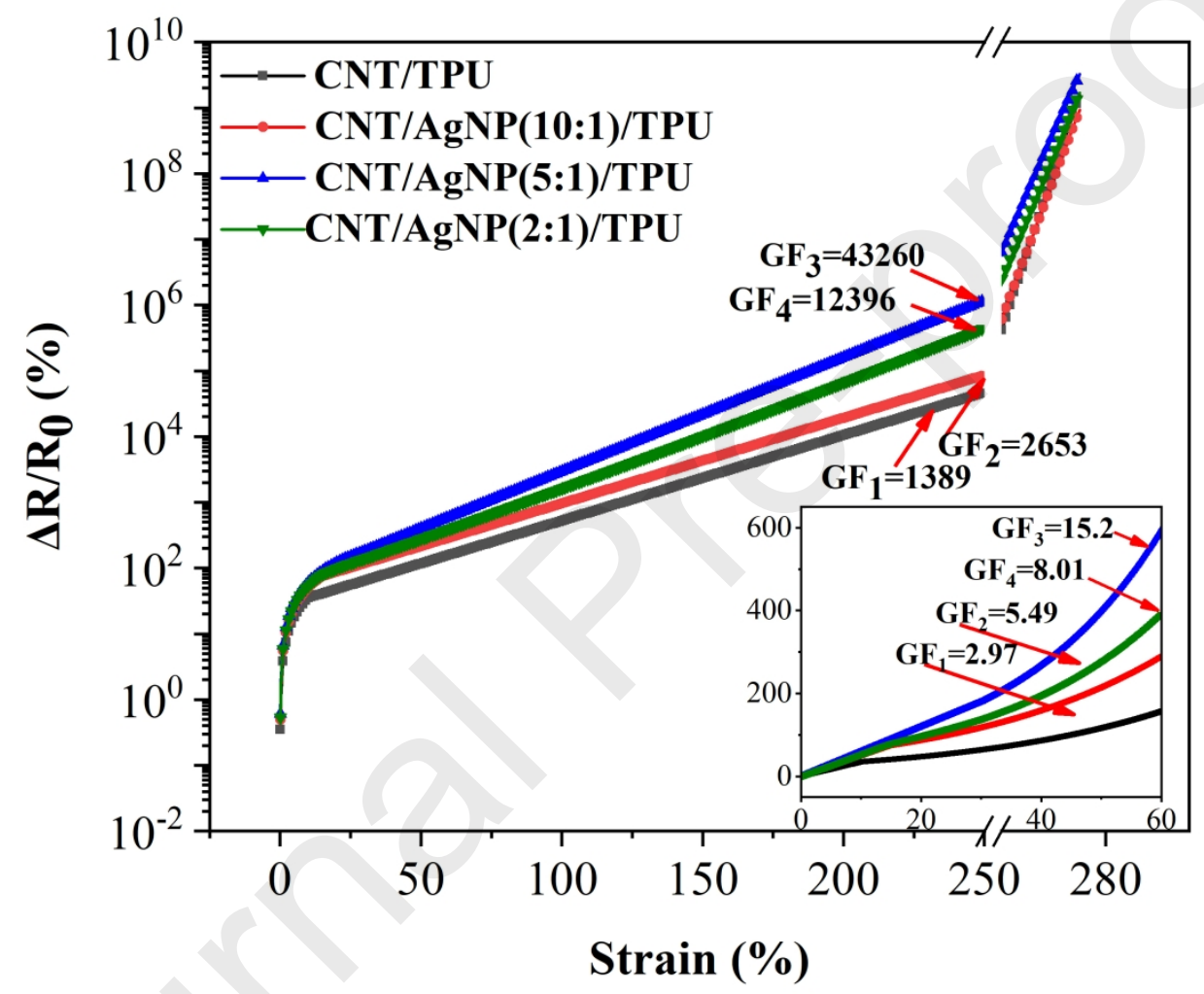

Fig. 7. Gauge factor of the printed strain sensors as a function of strain. 


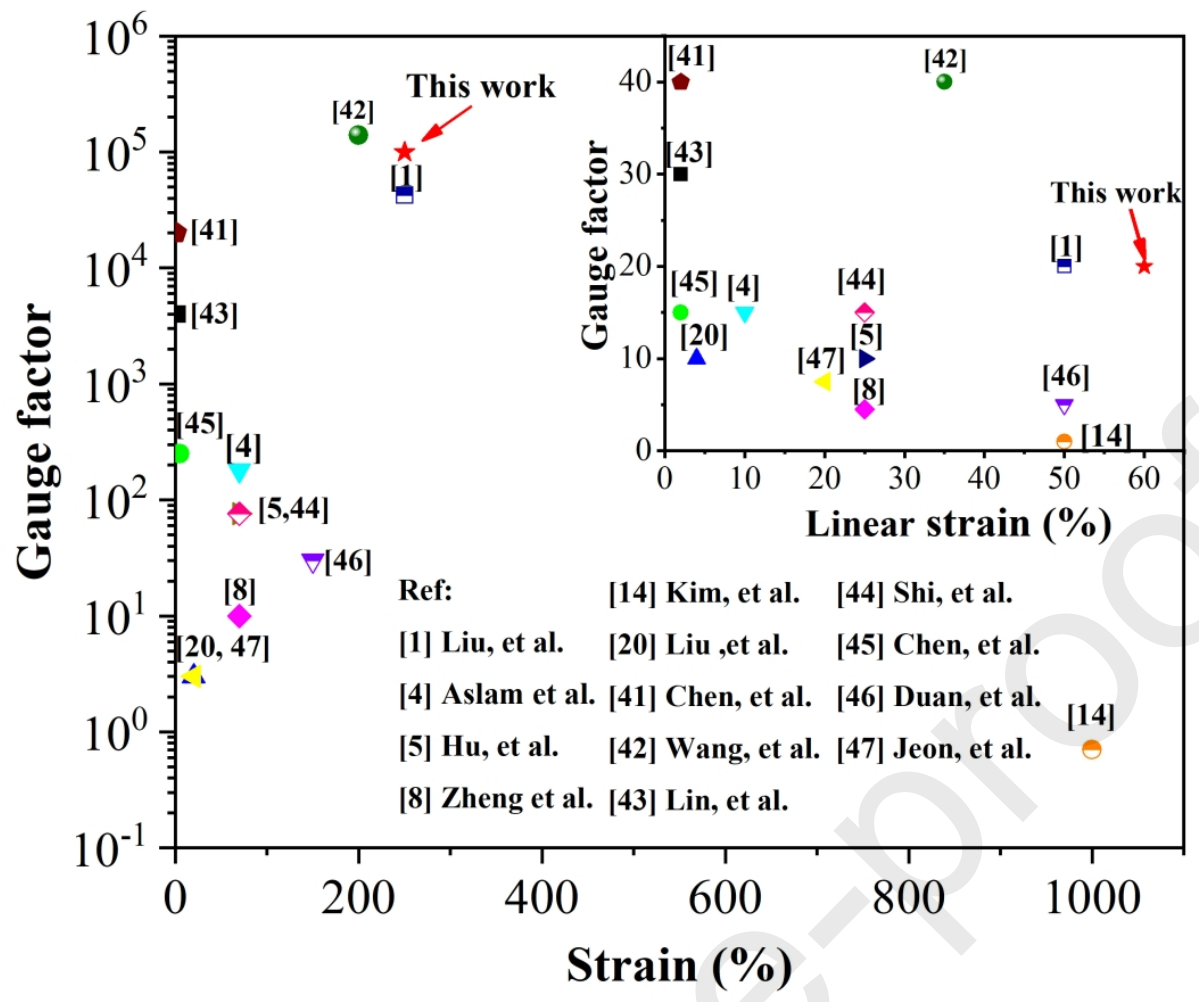

Fig. 8. Comparison of the gauge factor and the detectable strain range of the printed strain sensor with that of recently reported flexible strain sensors.

Fig. 9. The response time of the strain sensors with different CNT:AgNP weight ratios under cyclic loading/unloading processes: (a) CNT/TPU, (b) CNT/AgNP (10:1)/TPU, (c) CNT/AgNP (5:1)/TPU, (d) CNT/AgNP (2:1)/TPU.

The printed $\mathrm{CNT} / \operatorname{AgNP}(5: 1) / \mathrm{TPU}$ sensor was also investigated under cyclic loading/unloading at different strains with a frequency (v) of $0.1 \mathrm{~Hz}$. As shown in Fig. 10 , the $\Delta \mathrm{R} / \mathrm{R}_{0}$ of the sensor responded consistently to the loading/unloading cycles at strains $(\varepsilon)$ of $5 \%, 10 \%$, and $30 \%$, indicating that the sensor is capable of sensing multiple strain deformations. The $\Delta \mathrm{R} / \mathrm{R}_{0}$ of the strain sensor increased in tandem with the strain, which indicates the strain dependence of the sensor. The reason for the increase in the relative resistivity can be attributed to the increased damage to the conductive network with increasing strain.

To further investigate the effect of the CNT and AgNP content on the sensitivity of sensor under cyclic loading/unloading, the relative change in the resistance of the printed samples were compared at the same strain and frequency $(\varepsilon=30 \%, v=0.1)$, as shown in Fig. 11. It can be seen that CNT:AgNP(5:1)/TPU has the highest relative resistance change $\left(\Delta R / R_{0}=176\right)$ at the same loading conditions, which is consistent with the conclusion above in Fig.7. This indicates that the carbon nanotubes and silver nanoparticles have a more synergistic effect when CNT:AgNP $=5: 1$. The damage to the conductive network is sensitive to strain, and the sensitivity of the sensor is higher. 
(a)



(b)
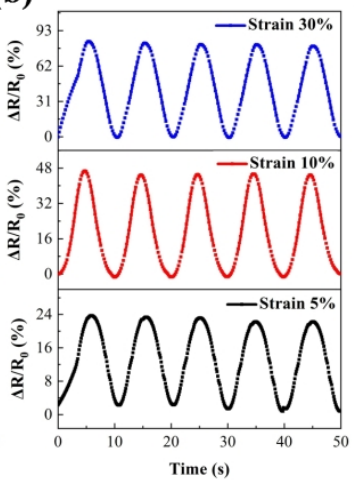

(c)


(d)
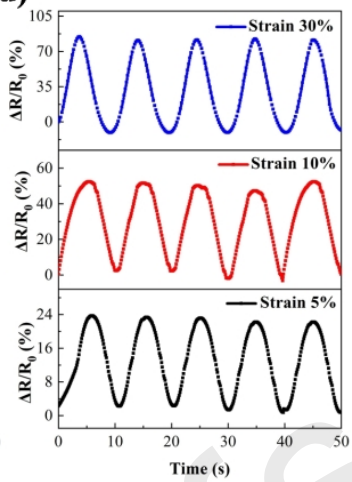

Fig. 10. Relative resistance change of the strain sensors with different CNT:AgNP weight ratios under cyclic loading/unloading processes at different strains $(5 \%, 10 \%$, $30 \%$ ) at a frequency of $0.1 \mathrm{~Hz}$ : (a) CNT/TPU, (b) CNT/AgNP (10:1)/TPU, (c) CNT/AgNP (5:1)/TPU, (d) CNT/AgNP (2:1)/TPU.



Fig. 11. Relative resistance changes of the strain sensors with different CNT:AgNP weight ratios at a cyclic strain of $30 \%$ and a frequency of $0.1 \mathrm{~Hz}$.

The relative resistance change of the CNT/TPU and CNT/AgNP(5:1)/TPU strain sensors at a cyclic strain of $30 \%$ and frequencies of $0.01,0.1,0.2$, and $1 \mathrm{~Hz}$ is shown in 
Fig. 12. All the sensors exhibit excellent responses over this wide testing frequency range. The $\Delta R / R_{0}$ of the sensors increases as the frequency increases, indicating a frequency dependence. The stiffer mechanical response on increasing the strain frequency is attributed to a reduction in the molecular mobility of the polymer chains [49].

Further cyclic stretching/releasing tests (up to 1000 cycles) were performed for the 3D printed strain sensors at a strain of $10 \%$ and a frequency of $1 \mathrm{~Hz}$ in order to examine the stability and repeatability of the strain sensing behavior, as shown in Fig. 13. As can be seen in Fig. 13a, the printed CNT/TPU sensor has a significant increase in resistance after 700 cycles. This indicates that the agglomeration of CNTs results in a conductive network structure in the TPU matrix that is more vulnerable to damage. Fig. 13b shows that the tested strain sensor $(\mathrm{CNT} / \operatorname{AgNP}(5: 1) / \mathrm{TPU})$ has good strain sensing stability even after 1000 test cycles. During the loading process, the nanofillers tend to reorient along the in-plane direction following the movement of the polymer chains, which leads to the continuous destruction and reconstruction of the conducting network [50,51]. The addition of AgNPs improved the dispersion of the nanoparticles in the TPU matrix and reduced agglomeration between the CNTs. These agglomerates are more susceptible to damage and reorientation during stretching, which results in reduced sensitivity, durability, and linearity of the sensor [50,52]. 
(a)

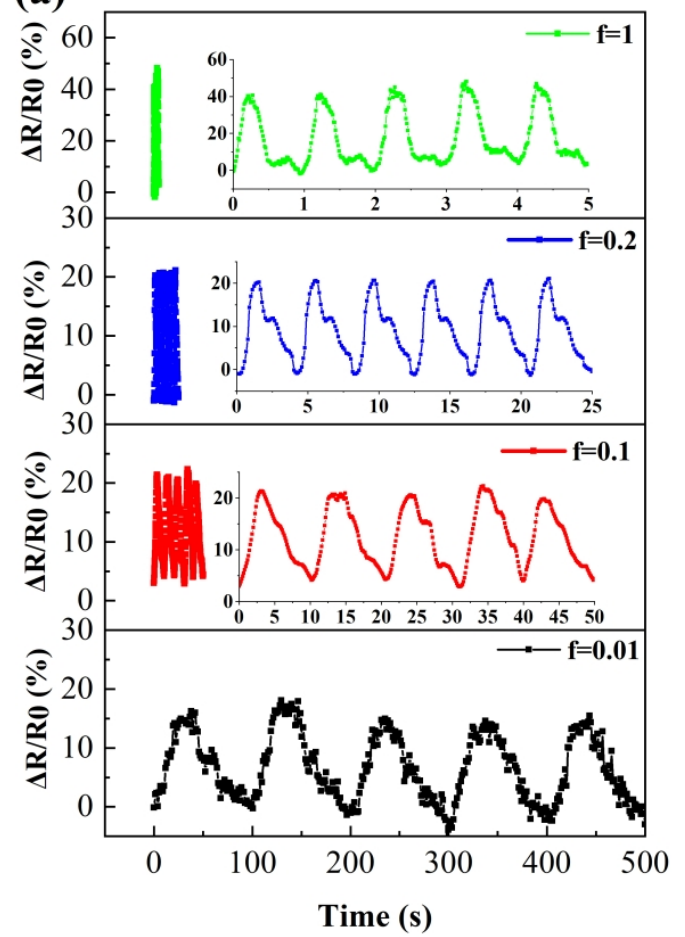

(b)

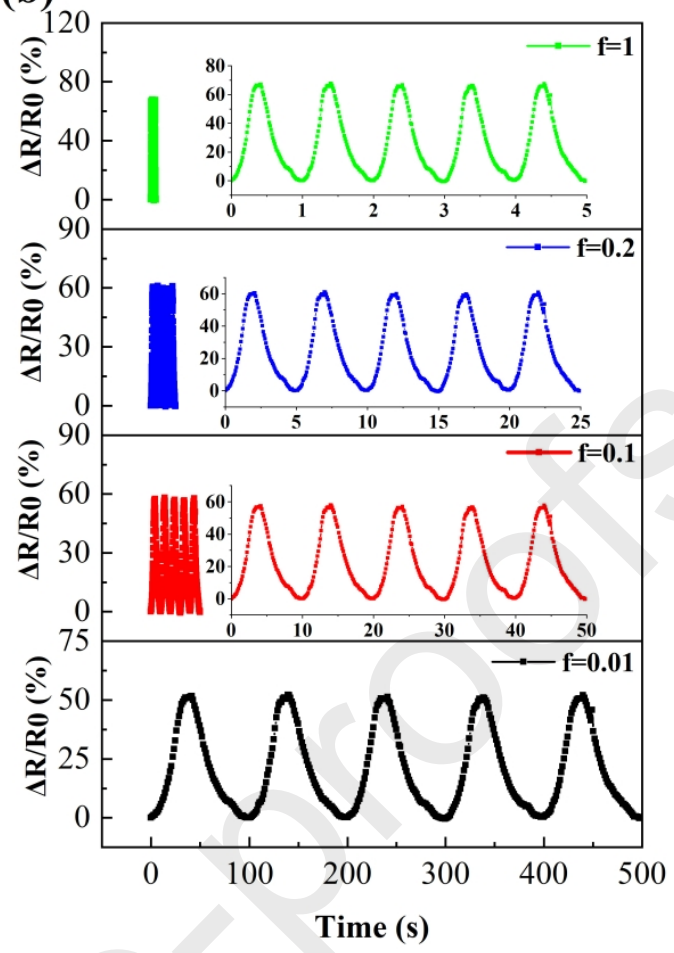

Fig. 12. Relative resistance change of the $3 \mathrm{D}$ printed strain sensors during cyclic loading at a strain of $10 \%$ and a frequency of $0.01,0.1,0.2$, and $1 \mathrm{~Hz}$ : (a) CNT/TPU, (b) CNT/AgNP(5:1)/TPU.
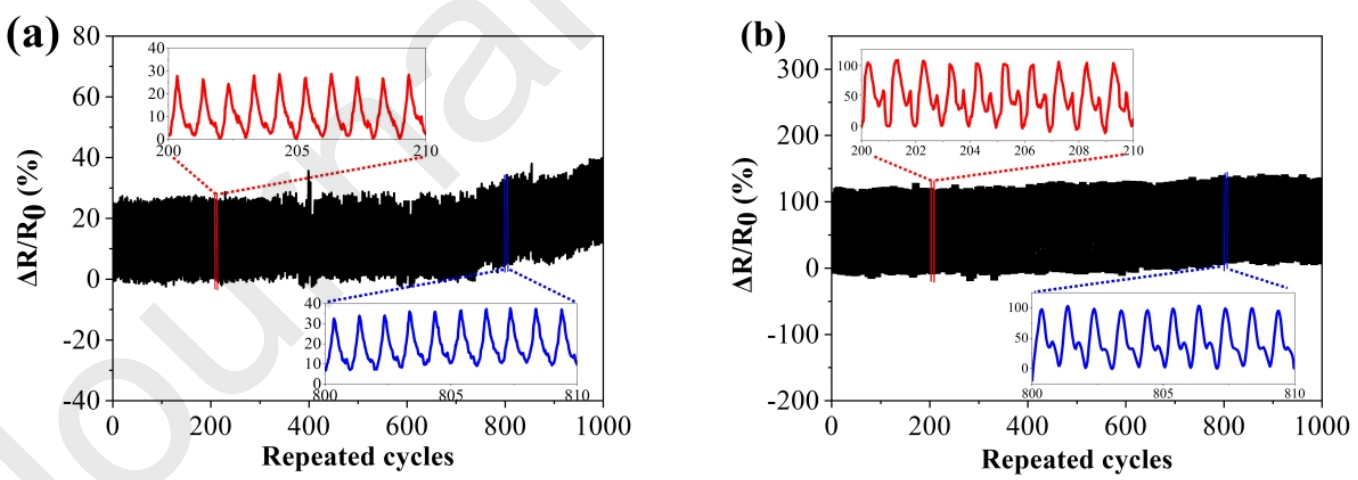

Fig. 13. Stability of the 3D printed strain sensors over 1000 cycles at a strain of $10 \%$ and a frequency of $0.1 \mathrm{~Hz}$ : (a) CNT/TPU, (b) CNT/TPU (5:1)/TPU.

\subsection{Modeling and mechanism}

Polymer/CNT nanocomposites exhibit a conducting behavior, which can be 
explained as the individual tunneling of electrons from the first CNT electrode to the next-nearest CNT electrode, forming a CNT/polymer pathway [53]. To better understand the sensing mechanism in printed sensors, modeling studies were carried out [54,55]. According to the model derived from tunneling theory by Simmons [42], the total resistance $\mathrm{R}$ of a composite can be calculated using Eq. (4):

$$
\begin{aligned}
& \mathrm{R}=R_{\text {tunnel }}+R_{\text {cn }}=\left(\frac{L}{N}\right)\left(\frac{8 \pi h s}{3 \gamma a^{2} e^{2}}\right) \exp (\gamma s) \\
& \gamma=\frac{4 \pi \sqrt{2 m \varphi}}{h}
\end{aligned}
$$

where $R_{c n}$ is the resistance of the CNTs, which is mainly affected by the conductivity and diameter of the CNTs, $R_{\text {tunneling }}$ is the tunneling resistance between two neighboring CNTs. $L$ is the number of particles that form a single conductive path, $N$ the number of conducting paths, $h$ the Planck's constant, s the shortest distance between conductive particles, $a^{2}$ the effective cross-section area, $e$ the electron charge, $m$ the electron mass, and $\varphi$ the height of the potential barrier between particles. Additionally, the CNTs can be modeled by a resistance network (Fig. 14). $R_{c n}$ is much less than $R_{\text {tunneling }}$ at a low filler content. At this point, the resistance of the CPCs is mainly determined by the tunneling effect, and the effect of $R_{c n}$ on the network is negligible. As the strain increases, both the tunneling distance and the change in conductive path markedly increase.

The application of strain to composite changes the resistance because of particle separation and the interparticle distance changes linearly and proportionally (for small strains) with increased strain from $s_{0}$ to $s$. This can be expressed as follows:

$$
\mathrm{s}=s_{0}\left(1+\mathrm{C}\left(\frac{\Delta l}{l_{\mathrm{o}}}\right)\right)=s_{0}\left(1+C_{\varepsilon}\right)
$$


where $\varepsilon$ is the tensile strain of the composites, $\Delta l$ is the deformation of the composites samples, and $l_{\mathrm{o}}$ is the initial length of the sample, and $C$ is a constant with different values in different material systems.

The non-linear rate of resistivity increase at larger applied strains can be represented by Eq. (7):

$$
\mathrm{N}=\frac{N_{\circ}}{\exp \left(M \varepsilon+W \varepsilon^{2}+U \varepsilon^{3}+V \varepsilon^{4}\right)}
$$

where M, W, U, and V are constants. Substitution of Eq. (7) and (6) into Eq. (4) gives Eq. (8):

$$
\mathrm{R}=\mathrm{B}(1+\mathrm{C} \varepsilon) \exp \left[A+(M+A C) \varepsilon+W \varepsilon^{2}+U \varepsilon^{3}+V \varepsilon^{4}\right.
$$

where $\mathrm{A}=\gamma S_{\mathrm{o}}, \mathrm{B}=\frac{8 \pi h n s_{\mathrm{o}}}{3 \gamma N_{\mathrm{o}}^{2} e^{2} a^{2}}$, and $\mathrm{n}$ is the total number of conductive particles $(\mathrm{n}=\mathrm{L}$ $\times \mathrm{N})$.

Fig. 15 shows that this equation models our experimental data well. The fitting parameters A, B, C, M, W, U, and V are listed in Table 5. Fig. 16a and b, show the change of the tunneling distance (change of $\mathrm{TD}, \mathrm{y}=\mathrm{Cx}$ ) and the number of conductive pathways (change of $\mathrm{CP}, \mathrm{y}=\mathrm{Mx}+\mathrm{Wx}^{2}+\mathrm{Ux}^{3}+\mathrm{Vx}^{4}$ ) against strain, respectively. The tunneling distance generally increases linearly with strain. Upon deformation, the number of conductive pathways in $\mathrm{CNT} / \operatorname{AgNP}(5: 1) / \mathrm{TPU}$ decreased more significantly than that in the CNT/AgNP(2:1)/TPU, and the CNT/TPU exhibits the lowest rate of change among all the tested samples. Additionally, the tunneling distance in the $\mathrm{CNT} / \operatorname{AgNP}(5: 1) / \mathrm{TPU}$ increases at a higher rate than that in the CNT/AgNP(2:1)/TPU and CNT/TPU. The change of TD and CP verify that the CPCs with CNT/AgNP(5:1) have a greater synergy between the hybrid nanofillers in the TPU matrix because the 
conductive network has fewer entanglements. This results in the conductive network deforming significantly under strain. This indicates that the effect of AgNPs on conductive network may be different for the samples with or without deformation. The AgNPs can bridge the CNTs to form more conductive pathways for the unstrained samples. However, when strain is applied to the CNT/AgNP/TPU samples, the destruction of conductive network is facilitated due to fewer entanglements.

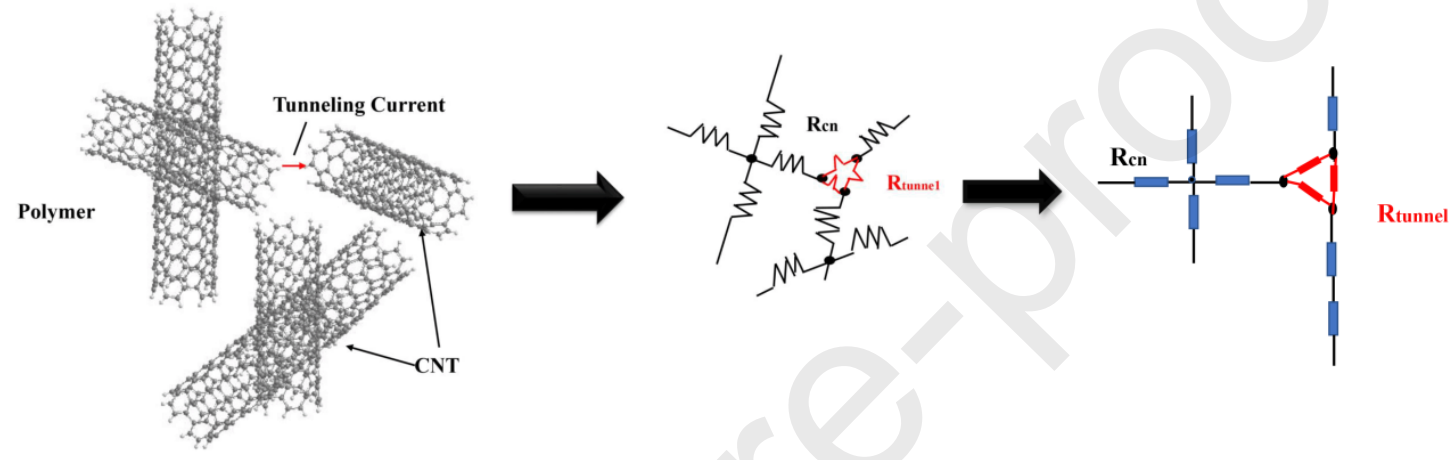

Fig. 14. Two-step modeling of the tunneling resistance in the resistor network.

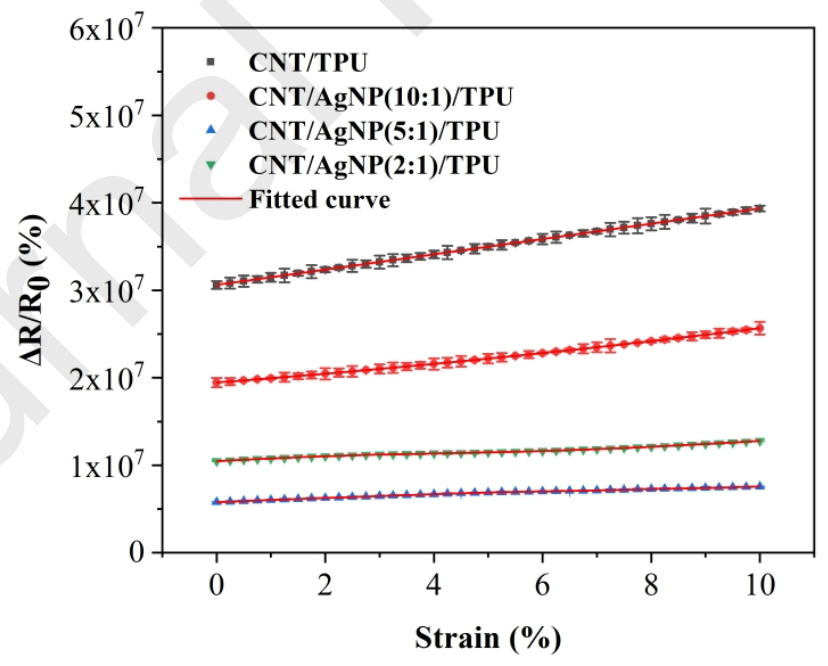

Fig. 15. Experimental (dots) and theoretical (red solid lines) results for the strain-resistance relationship of the printed sensors. 

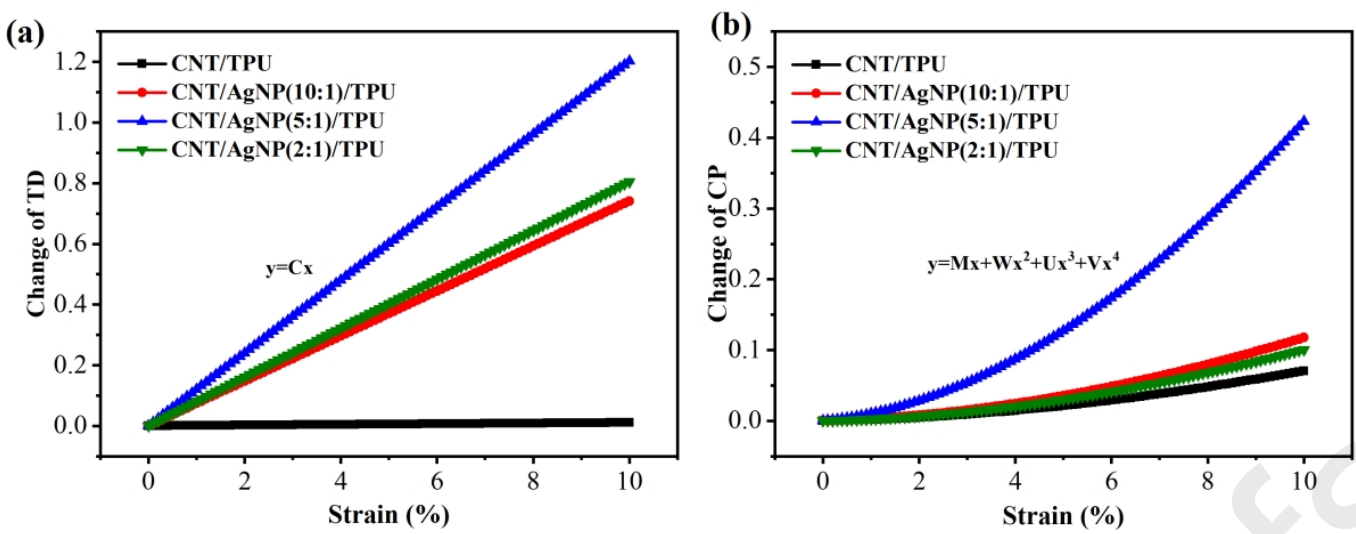

Fig. 16. Change of the (a) tunneling distance and (b) the conductive pathways as a function of strain for the strain sensors.

Table 5. Parameters obtained by fitting the strain-resistance curves of the printed sensors.

\begin{tabular}{|c|c|c|c|c|c|c|c|}
\hline Samples & $\mathbf{A}$ & B & $\mathbf{C}$ & $\mathbf{M}$ & $\mathbf{W}$ & $\mathbf{U}$ & $\mathbf{V}$ \\
\hline CNT/TPU & 0.5855 & $6.055 \times 10^{6}$ & 0.001070 & 0.004200 & $9.145 \times 10^{-4}$ & $-3.486 \times 10^{-6}$ & $-5.226 \times 10^{-8}$ \\
\hline CNT/AgNP(10:1)/TPU & 0.4866 & $9.714 \times 10^{6}$ & 0.07407 & 0.001232 & 0.001870 & $-1.847 \times 10^{-5}$ & $6.597 \times 10^{-8}$ \\
\hline CNT/AgNP(5:1)/TPU & 0.3210 & $1.441 \times 10^{5}$ & 0.1202 & 0.05124 & 0.003430 & $-4.410 \times 10^{-5}$ & $2.142 \times 10^{-7}$ \\
\hline CNT/AgNP(2:1)/TPU & 0.4336 & $1.851 \times 10^{7}$ & 0.07797 & 0.01127 & 0.001671 & $-1.759 \times 10^{-5}$ & $5.523 \times 10^{-7}$ \\
\hline
\end{tabular}

\subsection{Applications}

Because of their excellent sensitivity, linearity, detectable range, stability, and response speed, the printed $\mathrm{CNT} / \operatorname{AgNP}(5: 1) / \mathrm{TPU}$ strain sensor should have excellent potential for use in wearable medical devices etc. A number of potential applications including human motion detection such as finger movements and bending at the wrist were evaluated and the results are shown in Fig. 17. When the strain sensor was attached to the index finger, bending of the finger could clearly be detected, with 
different responses for various bending degrees (Fig. 17a). For example, the $\Delta R / R_{0}$ reached $5 \%$ at a bending angle of $30^{\circ}$, and it increased to $20 \%$ at a bending angle of $120^{\circ}$. The change of the relative resistivity was proportional, which further indicated that the printed strain sensor had a high linearity. Fig. 17b shows the response of the strain sensor to wrist bending. The $\Delta \mathrm{R} / \mathrm{R}_{0}$ response was $7.5 \%$ at a bending angle of $30^{\circ}$, and this increased to $28.3 \%$ at a bending angle of $90^{\circ}$. The sensor was then used to detect the opening of the mouth. For this, the flexible sensor was attached to the cheek (Fig. 17c). In addition to these large movements, other small-scale human physiological activities were also recorded. As shown in Fig. 17d, our printed sensor could be utilized to monitor the swallowing process. When a volunteer swallowed, the sensors deformed because of the muscle movement near the esophagus, and there was a significant corresponding resistance change of the sensor due to its high sensitivity. In addition, the resistance of the printed sensor is almost not changed at $20-60{ }^{\circ} \mathrm{C}$ (Fig. S1 in Supporting Information), indicating a wide operating temperature for monitoring human and robot motions etc. 

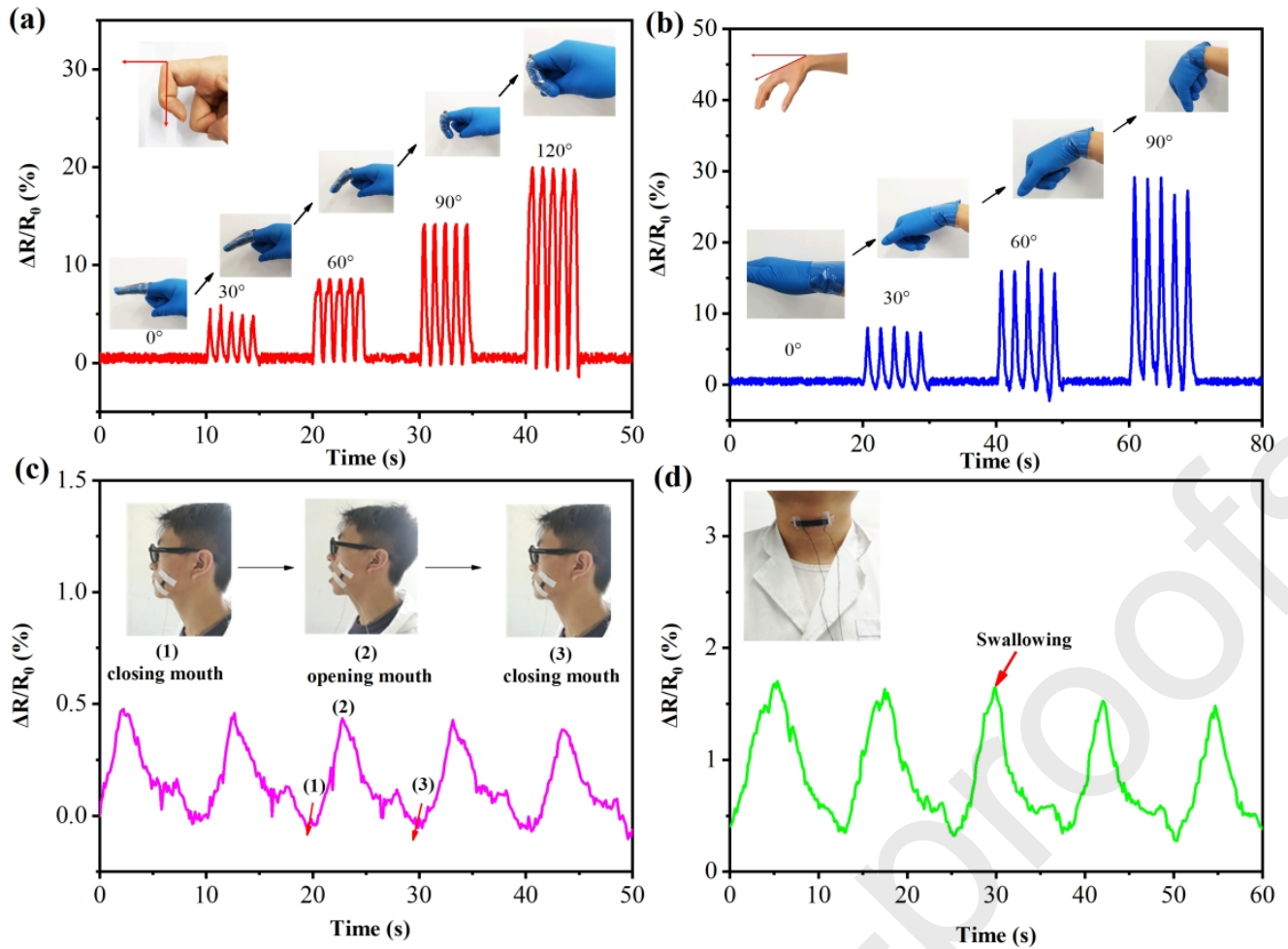

Fig. 17. Electromechanical responses of the printed sensor to repeated bending and unbending of (a) index finger and (b) wrist, (c) mouth opening, and (d) swallowing.

\section{Conclusions}

In this study, high-elastic strain sensors of ternary CNT/AgNP/TPU composites were prepared via 3D printing, and AgNPs were synthesized in the presence of CNTs and introduced to improve the electrical and sensing properties of the composites. The main reason for introducing the AgNPs in this study was to reduce the interaction (entanglement potential) between neighboring CNTs and also to tailor the conductive pathways between the composites comprised of AgNPs and CNTs. Compared with the printed CNT/TPU nanocomposite, the resistivity of the CNT:AgNP(5:1)/TPU decreased 2.58 fold. The sensor exhibited a high sensitivity (GF $=43260$ at a strain of $250 \%$ for 
the printed CNT:AgNP (5:1)/TPU sensor), a wide detectable strain range (up to $250 \%$ ) and a linearity close to 1. Additionally, this sensor exhibited regular frequency responses within the test frequency range $(0.01-1 \mathrm{~Hz})$ indicating the potential for monitoring frequency changes. Furthermore, this sensor exhibited excellent stability and repeatability during cyclic strain testing for up to 1000 cycles. Based on tunneling theory, the strain sensor mechanism was modeled and the experimental data was in good agreement with the theoretical equation. The ability of the sensor to monitor human motions, including finger movements, joint movements, mouth opening, and swallowing was also demonstrated. This work provides guidelines for the $3 \mathrm{D}$ printing of high-performance flexible strain sensors with potential applications in intelligent robots, prosthetics, and wearable devices where complex design and customizability are required.

\section{Acknowledgements}

This work is supported by Sichuan Science and Technology Program (2017HH0086, 2017JY0152), Undergraduate Innovation Program of Sichuan Province (201910615027), and Scientific Research Foundation for the Returned Overseas Chinese Scholars of Sichuan Province.

\section{References}

[1] Liu S, Li L. Ultrastretchable and Self-Healing Double-Network Hydrogel for 3D Printing and Strain Sensor. ACS Appl Mater Interfaces 2017;9:26429-26437. doi:10.1021/acsami.7b07445. 
[2] Hassan G, Bae J, Hassan A, Ali S, Lee CH, Choi Y. Ink-jet printed stretchable strain sensor based on graphene/ZnO composite on micro-random ridged PDMS substrate. Compos Part A Appl Sci Manuf 2018;107:519-528.

doi:10.1016/j.compositesa.2018.01.031.

[3] Shi J, Li X, Cheng H, Liu Z, Zhao L, Yang T, et al. Graphene Reinforced Carbon Nanotube Networks for Wearable Strain Sensors. Adv Funct Mater 2016;26:2078-2084. doi:10.1002/adfm.201504804.

[4] Aslam S, Bokhari TH, Anwar T, Khan U, Nairan A, Khan K. Graphene oxide coated graphene foam based chemical sensor. Mater Lett 2019;235:66-70. doi:10.1016/j.matlet.2018.09.164.

[5] Hu C, Li Z, Wang Y, Gao J, Dai K, Zheng G, et al. Comparative assessment of the strain-sensing behaviors of polylactic acid nanocomposites: reduced graphene oxide or carbon nanotubes. J Mater Chem C 2017;5:2318-2328. doi:10.1039/c6tc05261d.

[6] Ma Y, Liu N, Li L, Hu X, Zou Z, Wang J, et al. A highly flexible and sensitive piezoresistive sensor based on MXene with greatly changed interlayer distances. Nat Commun 2017;8:1-7. doi:10.1038/s41467-017-01136-9.

[7] Jeon JY, Ha TJ. Waterproof Electronic-Bandage with Tunable Sensitivity for Wearable Strain Sensors. ACS Appl Mater Interfaces 2016;8:2866-71. doi:10.1021/acsami.5b12201.

[8] Zheng Q, Liu X, Xu H, Cheung MS, Choi YW, Huang HC, et al. Sliced graphene foam films for dual-functional wearable strain sensors and switches. Nanoscale Horizons 2018;3:35-44. doi:10.1039/c7nh00147a.

[9] Liu T, Tian X, Zhang M, Abliz D, Li D, Ziegmann G. Interfacial performance and fracture patterns of 3D printed continuous carbon fiber with sizing reinforced PA6 composites. Compos Part A Appl Sci Manuf 2018;114:368-376. doi:10.1016/j.compositesa.2018.09.001.

[10] Liu J, Li W, Guo Y, Zhang H, Zhang Z. Improved thermal conductivity of thermoplastic polyurethane via aligned boron nitride platelets assisted by $3 \mathrm{D}$ printing. Compos Part A Appl Sci Manuf 2019;120:140-146. doi:10.1016/j.compositesa.2019.02.026.

[11] Ryu S, Lee P, Chou JB, Xu R, Zhao R, Hart AJ, et al. Extremely Elastic Wearable Carbon Nanotube Fiber Strain Sensor for Monitoring of Human Motion. ACS Nano 2015;9:5929-5936. doi:10.1021/acsnano.5b00599.

[12] Liu Y, Hou C, Jiao T, Song J, Zhang X, Xing R, et al. Self-Assembled AgNP-Containing Nanocomposites Constructed by Electrospinning as Efficient Dye Photocatalyst Materials for Wastewater Treatment. Nanomaterials 2018;8:35. doi:10.3390/nano8010035.

[13] Wei Y, Chen S, Dong X, Lin Y, Liu L. Flexible piezoresistive sensors based on "dynamic bridging effect" of silver nanowires toward graphene. Carbon 2017;113:395-403. doi:10.1016/j.carbon.2016.11.027.

[14] Kim K, Park J, Suh J hoon, Kim M, Jeong Y, Park I. 3D printing of multiaxial force sensors using carbon nanotube (CNT)/thermoplastic polyurethane (TPU) filaments. Sensors Actuators, A Phys 2017;263:493-500. 
doi:10.1016/j.sna.2017.07.020.

[15] Jia Y, Chen Z, Yan W. A numerical study on carbon nanotube pullout to understand its bridging effect in carbon nanotube reinforced composites. Compos Part B Eng 2015;81:64-71. doi:10.1016/j.compositesb.2015.07.003.

[16] Liu H, Gao J, Huang W, Dai K, Zheng G, Liu C, et al. Electrically conductive strain sensing polyurethane nanocomposites with synergistic carbon nanotubes and graphene bifillers. Nanoscale 2016;8:12977-12989. doi:10.1039/c6nr02216b.

[17] Cai W, Huang Y, Wang D, Liu C, Zhang Y. Piezoresistive behavior of graphene nanoplatelets/carbon black/silicone rubber nanocomposite. J Appl Polym Sci 2014;131:1-6. doi:10.1002/app.39778.

[18] Ma Z, Wei A, Ma J, Shao L, Jiang H, Dong D, et al. Lightweight, compressible and electrically conductive polyurethane sponges coated with synergistic multiwalled carbon nanotubes and graphene for piezoresistive sensors. Nanoscale 2018;10:7116-126. doi:10.1039/c8nr00004b.

[19] Sagalianov I, Vovchenko L, Matzui L, Lazarenko O. Synergistic Enhancement of the Percolation Threshold in Hybrid Polymeric Nanocomposites Based on Carbon Nanotubes and Graphite Nanoplatelets. Nanoscale Res Lett 2017;12:1-7. doi:10.1186/s11671-017-1909-z.

[20] Liu X, Tang C, Du X, Xiong S, Xi S, Liu Y, et al. A highly sensitive graphene woven fabric strain sensor for wearable wireless musical instruments. Mater Horizons 2017;4:477-86. doi:10.1039/c7mh00104e.

[21] Liu W, Wu N, Pochiraju K. Shape recovery characteristics of SiC/C/PLA composite filaments and 3D printed parts. Compos Part A Appl Sci Manuf 2018;108:1-11. doi:10.1016/j.compositesa.2018.02.017.

[22] Kwon J, Cho H, Eom H, Lee H, Suh YD, Moon H, et al. Low-Temperature Oxidation-Free Selective Laser Sintering of $\mathrm{Cu}$ Nanoparticle Paste on a Polymer Substrate for the Flexible Touch Panel Applications. ACS Appl Mater Interfaces 2016;8:11575-11582. doi:10.1021/acsami.5b12714.

[23] Ota H, Emaminejad S, Gao Y, Zhao A, Wu E, Challa S, et al. Application of 3D Printing for Smart Objects with Embedded Electronic Sensors and Systems. Adv Mater Technol 2016;1:1600013. doi:10.1002/admt.201600013.

[24] Kim JH, Lee S, Wajahat M, Jeong H, Chang WS, Jeong HJ, et al. Three-Dimensional Printing of Highly Conductive Carbon Nanotube Microarchitectures with Fluid Ink. ACS Nano 2016;10:8879-8887. doi:10.1021/acsnano.6b04771.

[25] Zhang Q, Zhang F, Xu X, Zhou C, Lin D. Three-Dimensional Printing Hollow Polymer Template-Mediated Graphene Lattices with Tailorable Architectures and Multifunctional Properties. ACS Nano 2018;12:1096-1106. doi:10.1021/acsnano.7b06095.

[26] Duan S, Yang K, Wang Z, Chen M, Zhang L, Zhang H, et al. Fabrication of Highly Stretchable Conductors Based on 3D Printed Porous Poly(dimethylsiloxane) and Conductive Carbon Nanotubes/Graphene Network. ACS Appl Mater Interfaces 2016;8:2187-2192. doi:10.1021/acsami.5b10791. 
[27] Gnanasekaran K, Heijmans T, van Bennekom S, Woldhuis H, Wijnia S, de With $\mathrm{G}$, et al. 3D printing of CNT- and graphene-based conductive polymer nanocomposites by fused deposition modeling. Appl Mater Today 2017;9:21-28. doi:10.1016/j.apmt.2017.04.003.

[28] Christ JF, Aliheidari N, Ameli A, Pötschke P. 3D printed highly elastic strain sensors of multiwalled carbon nanotube/thermoplastic polyurethane nanocomposites. Mater Des 2017;131:394-401. doi:10.1016/j.matdes.2017.06.011.

[29] Yoon Y, Samanta K, Lee H, Lee K, Tiwari AP, Lee J, et al. Highly Stretchable and Conductive Silver Nanoparticle Embedded Graphene Flake Electrode Prepared by In situ Dual Reduction Reaction. Sci Rep 2015;5:1-10. doi:10.1038/srep14177.

[30] Poudel BK, Park JH, Byeon JH. On-Demand Gas-to-Liquid Process To Fabricate Thermoresponsive Antimicrobial Nanocomposites and Coatings. ACS Appl Mater Interfaces 2017;9:15342-15349. doi:10.1021/acsami.7b05167.

[31] Dou Y, Liu H, Peng J, Li M, Li W, Yang F. A green method for preparation of $\mathrm{CNT/CS} / \mathrm{AgNP}$ composites and evaluation of their catalytic performance. $\mathrm{J}$ Mater Sci 2016;51:5685-5694. doi:10.1007/s10853-016-9871-1.

[32] Subbiah R, Veerapandian M, Sadhasivam S, Yun K. Triad CNT-NPs/polymer nanocomposites: Fabrication, characterization, and preliminary antimicrobial study. Synth React Inorganic, Met Nano-Metal Chem 2011;41:345-355. doi:10.1080/15533174.2011.555868.

[33] Zhang X, Zhang J, Quan J, Wang N, Zhu Y. Surface-enhanced Raman scattering activities of carbon nanotubes decorated with silver nanoparticles. Analyst 2016;141:5527-5534. doi:10.1039/c6an00850j.

[34] Hohimer C, Aliheidari N, Mo C, Ameli A. Mechanical Behavior of 3D Printed Multiwalled Carbon Nanotube/Thermoplastic Polyurethane Nanocomposites. Vol 1 Dev Charact Multifunct Mater Mech Behav Act Mater Bioinspired Smart Mater Syst Energy Harvest Emerg Technol 2017:V001T08A004. doi:10.1115/SMASIS2017-3808.

[35] Lu Y, Jiang J, Yoon S, Kim KS, Kim JH, Park S, et al. High-Performance Stretchable Conductive Composite Fibers from Surface-Modified Silver Nanowires and Thermoplastic Polyurethane by Wet Spinning. ACS Appl Mater Interfaces 2018;10:2093-2104. doi:10.1021/acsami.7b16022.

[36] Tiwari JN, Vij V, Kemp KC, Kim KS. Engineered carbon-nanomaterial-based electrochemical sensors for biomolecules. ACS Nano 2016;10:46-80. doi:10.1021/acsnano.5b05690.

[37] Zheng Y, Li Y, Li Z, Wang Y, Dai K, Zheng G, et al. The effect of filler dimensionality on the electromechanical performance of polydimethylsiloxane based conductive nanocomposites for flexible strain sensors. Compos Sci Technol 2017;139:64-73. doi:10.1016/j.compscitech.2016.12.014.

[38] Amjadi M, Yoon YJ, Park I. Ultra-stretchable and skin-mountable strain sensors using carbon nanotubes-Ecoflex nanocomposites. Nanotechnology 2015;26:375501. doi:10.1088/0957-4484/26/37/375501. 
[39] Wu H, Liu Q, Chen H, Shi G, Li C. Fibrous strain sensor with ultra-sensitivity, wide sensing range, and large linearity for full-range detection of human motion. Nanoscale 2018;10:17512-1719. doi:10.1039/c8nr05404e.

[40] Cao X, Wei X, Li G, Hu C, Dai K, Guo J. Strain sensing behaviors of epoxy nanocomposites with carbon nanotubes under cyclic deformation. Polymer (Guildf) 2017;112:1-9. doi:10.1016/j.polymer.2017.01.068.

[41] Chen Q, Xiang D, Wang L, Tang Y, Harkin-Jones E, Zhao C, et al. Facile fabrication and performance of robust polymer/carbon nanotube coated spandex fibers for strain sensing. Compos Part A Appl Sci Manuf 2018;112:186-196. doi:10.1016/j.compositesa.2018.06.009.

[42] Wang J, Wang W, Zhang C, Yu W. The electro-mechanical behavior of conductive filler reinforced polymer composite undergone large deformation: A combined numerical-analytical study. Compos Part B Eng 2018;133:185-192. doi:10.1016/j.compositesb.2017.09.041.

[43] Lin L, Liu S, Zhang Q, Li X, Ji M, Deng H, et al. Towards tunable sensitivity of electrical property to strain for conductive polymer composites based on thermoplastic elastomer. ACS Appl Mater Interfaces 2013;5:5815-5824. doi:10.1021/am401402x.

[44] Shi J, Hu J, Dai Z, Zhao W, Liu P, Zhao L, et al. Graphene welded carbon nanotube crossbars for biaxial strain sensors. Carbon 2017;123:786-793. doi:10.1016/j.carbon.2017.08.006.

[45] Chen S, Wei Y, Wei S, Lin Y, Liu L. Ultrasensitive Cracking-Assisted Strain Sensors Based on Silver Nanowires/Graphene Hybrid Particles. ACS Appl Mater Interfaces 2016;8:25563-25570. doi:10.1021/acsami.6b09188.

[46] Duan L, Fu S, Deng H, Zhang Q, Wang K, Chen F, et al. The resistivity-strain behavior of conductive polymer composites: Stability and sensitivity. J Mater Chem A 2014;2:17085-17098. doi:10.1039/c4ta03645j.

[47] Jeon H, Hong SK, Kim MS, Cho SJ, Lim G. Omni-Purpose Stretchable Strain Sensor Based on a Highly Dense Nanocracking Structure for Whole-Body Motion Monitoring. ACS Appl Mater Interfaces 2017;9:41712-41721. doi:10.1021/acsami.7b14153.

[48] Ge G, Cai Y, Dong Q, Zhang Y, Shao J, Huang W, et al. A flexible pressure sensor based on $\mathrm{rGO}$ /polyaniline wrapped sponge with tunable sensitivity for human motion detection. Nanoscale 2018;10:10033-10040. doi:10.1039/c8nr02813c.

[49] Lozano-Pérez C, Cauich-Rodríguez J V., Avilés F. Influence of rigid segment and carbon nanotube concentration on the cyclic piezoresistive and hysteretic behavior of multiwall carbon nanotube/segmented polyurethane composites. Compos Sci Technol 2016;128:25-32. doi:10.1016/j.compscitech.2016.03.010.

[50] Hou Y, Wang D, Zhang XM, Zhao H, Zha JW, Dang ZM. Positive piezoresistive behavior of electrically conductive alkyl-functionalized graphene/polydimethylsilicone nanocomposites. J Mater Chem C 2013;1:515521. doi:10.1039/c2tc00114d.

[51] Fernández-d'Arlas B, Khan U, Rueda L, Coleman JN, Mondragon I, Corcuera 
MA, et al. Influence of hard segment content and nature on polyurethane/multiwalled carbon nanotube composites. Compos Sci Technol 2011;71:1030-1038. doi:10.1016/j.compscitech.2011.02.006.

[52] Gong S, Zhu ZH. Modeling sensor behavior of CNT/polymer nanocomposite. 14th IEEE Int Conf Nanotechnology, IEEE-NANO 2014 2014:1029-1034. doi:10.1109/NANO.2014.6968167.

[53] Zhang X, Pan YI, Zheng Q, Yi X. Time Dependence of Piezoresistance for the Conductor-. J Appl Polym Sci 2000;38:2739-2749.

[54] Hu N, Karube Y, Yan C, Masuda Z, Fukunaga H. Tunneling effect in a polymer/carbon nanotube nanocomposite strain sensor. Acta Mater 2008;56:2929-2936. doi:10.1016/j.actamat.2008.02.030.

[55] Liu C, Huang N, Xu F, Tong J, Chen Z, Gui X, et al. 3D printing technologies for flexible tactile sensors toward wearable electronics and electronic skin. Polymers (Basel) 2018;10:1-31. doi:10.3390/polym10060629. 